\title{
SUN2: A potential therapeutic target in cancer (Review)
}

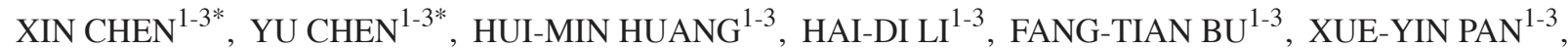 \\ YANG YANG ${ }^{1-3}$, WAN-XIA LI ${ }^{4}$, XIAO-FENG LI ${ }^{1-3}$, CHENG HUANG $^{1-3}$, XIAO-MING MENG $^{1-3}$ and JUN LI ${ }^{1-3}$ \\ ${ }^{1}$ School of Pharmacy, Anhui Medical University; ${ }^{2}$ The Key Laboratory of Anti-inflammatory and Immune Medicines, \\ Ministry of Education; ${ }^{3}$ Institute for Liver Diseases of Anhui Medical University, Hefei, Anhui 230032; \\ ${ }^{4}$ Department of Pharmacy, Anqing Municipal Hospital, Anqing, Anhui 246003, P.R. China
}

Received August 8, 2017; Accepted April 30, 2018

DOI: $10.3892 / \mathrm{ol} .2018 .9764$

\begin{abstract}
The incidence of cancer is increasing at an alarming rate despite recent advances in prevention strategies, diagnostics and therapeutics for various types of cancer. The identification of novel biomarkers to aid in prognosis and treatment for cancer is urgently required. Uncontrolled proliferation and dysregulated apoptosis are characteristics exhibited by cancer cells in the initiation of various types of cancer. Notably, aberrant expression of crucial oncogenes or cancer suppressors is a defining event in cancer occurrence. Research has demonstrated that SAD1/UNC84 domain protein-2 (SUN2) serves a suppressive role in breast cancer, atypical teratoid/rhabdoid tumors and lung cancer progression. Furthermore, SUN2 inhibits cancer cell proliferation, migration and promotes apoptosis. Recent reports have also shown that SUN2 serves prominent roles in resistance to the excessive DNA damage that destabilizes the genome and promotes cancer development, and these functions of SUN2 are critical for evading
\end{abstract}

Correspondence to: Professor Jun Li, School of Pharmacy, Anhui Medical University, 81 Mei Shan Road, Hefei, Anhui 230032, P.R. China

E-mail: lj@ahmu.edu.cn

${ }^{*}$ Contributed equally

Abbreviations: SUN2, SAD1/UNC84 domain protein-2; LINC linker of nucleoskeleton and cytoskeleton; MEFs, mouse embryonic fibroblasts; KASH, klarsicht/ANC-1/syne-1 homology; AT/RT, atypical teratoid/rhabdoid tumor; OS, overall survival; DDR, DNA damage response; ATM, ataxia telangiectasia mutated; HU, hydroxyurea; 3'UTR, 3'-untranslated region; INM, inner nuclear membrane; ONM, outer nuclear membrane, MB, medulloblastoma; CDS, complete coding sequence; SIRT5, silent information regulator-5; HDAC, histone deacetylase; NSCLC, non-small cell lung cancer; GLUT1, glucose transporter-1; LDHA, lactate dehydrogenase A; PARP, poly (ADP-ribose) polymerase; JAK, janus protein tyrosine kinase; PTEN, phosphatase and tensin homology deleted on chromosome; PI3K, phosphatidylinositol 3-kinase; AKT, protein kinase B; DNA-PKcs, DNA-dependent protein kinase catalytic subunit

Key words: SUN2, cancer, proliferation, apoptosis, DNA damage initiation of cancer. Additionally, increasing evidence has demonstrated that SUN2 is involved in maintaining cell nuclear structure and appears to be a central component for organizing the natural nuclear architecture in cancer cells. The focus of the present review is to provide an overview on the pharmacological functions of SUN2 in cancers. These findings suggest that SUN2 may serve as a promising therapeutic target and novel predictive marker in various types of cancer.

\section{Contents}

1. Introduction

2. Structure of SUN2

3. The role of SUN2 in different cancers

4. SUN2 and DNA damage

5. Overview of SUN2 signaling pathways in cancer

6. Conclusion and prospective

\section{Introduction}

Cancers originate from normal cells that gain the ability to aberrantly proliferate and eventually turn malignant. These cancerous cells then grow clonally into tumors and eventually acquire the potential to metastasize (1). Alteration of cellular processes is a central component in cancer development, including changes in cancer cell growth, apoptosis, migration and invasion (2-6). Inhibition of the abnormal growth of cancer cells and the promotion of cancer cell apoptosis are widely recognized as crucial goals for intervention of cancer progression. In addition, dysregulations of oncogenes and cancer suppressors tightly correlate with cancer occurrence (7-9). However, the lack of useful cancer biomarkers and targets is a major contributor to the high mortality rate and prevalence of cancer.

SAD1/UNC84 domain protein-2 (SUN2), a member of the SUN domain protein family, is a key component of linker of nucleoskeleton and cytoskeleton (LINC) complex. The nuclear architecture functionally provides a framework for organizing and regulating diverse processes within cells. Notably, cancer cells generally exhibit variety of features indicative of atypical nuclei (10), although the molecular mechanism of these phenomena remains to be elucidated. A number of studies 
have shown that loss of LINC complexes reduces nuclear and cellular rigidity, increasing tissue fluidity, promoting invasive activity, and inducing cancer progression (10-12). Interestingly, studies have uncovered a fundamental role of SUN2 in nuclear structure determination function (13). Therefore, we hypothesize that the effects of SUN2 on regulating nuclear architecture may affect biological function in cancer cells. Indeed, abnormal expression of SUN2 and LINC complexes is associated with the occurrence of many human diseases, especially cancers (10).

Several studies have also linked SUN2 function with various cancers. SUN2 plays SUN2 also plays a cancer suppressor role in miR-221/222-mediated malignant embryonal tumors of the central nervous system (14). Another study confirmed that expression of SUN2 was reduced in breast cancer (10). Moreover, SUN2 exhibited suppression of lung cancer cell proliferation and migration and promotion of lung cancer cell apoptosis; SUN2 also enhanced the chemotherapy sensitivity of lung cancer cells exposed to cisplatin, and higher SUN2 level predicts a better overall survival in lung cancer progression (12). Together these studies indicate that dysregulation of SUN2 may be involved in cancer development.

Failure to detect and repair DNA damage leads to genomic instability, which is one of the hallmarks that drive cancer occurrence (15). Recent reports also suggest that SUN2 exhibits resistance to DNA damage and maintaining the genome integrity (16). Lei et al confirmed that SUN2 is required for attenuating excessive DNA damage in mouse embryonic fibroblasts (MEFs) from SUN1 ${ }^{-/}$SUN2 ${ }^{-/-}$double knockout mice (16). Whether SUN2 participates in maintaining genomic stability in other types of cancer needed further validation.

Following recent advances, this review presents recent information regarding the functions of SUN2 in the progression of cancer and discusses the emerging signal pathways regulated by SUN2 in cancer.

\section{Structure of SUN2}

The LINC complex is a nuclear envelope protein complex that mainly consists of SUN and nesprin proteins, connecting nuclear lamina and cytoskeletal filaments (10), LINC complex characteristics of architecture framework helps to regulate the size and shape of the cell nucleus. Several SUN proteins have been identified in several organisms, including Schizosaccharomyces pombe Sad1, Caenorhabditis elegans UNC-84 and SUN1, and five human SUN proteins (17). Human SUN proteins can be grouped into two subfamilies based on their intracellular localization: SUN1 and SUN2 are integral membrane components of the inner nuclear membrane (INM) (18-20); SUN3 and the sperm-associated antigen 4 localize to endoplasmic reticulum and outer nuclear membrane (ONM) $(21,22)$.

SUN proteins are conserved among all eukaryotes and characterized by a C-terminal 200 amino acid SUN domain (18,19,23-25). SUN proteins form a trimer through the SUN domain and exhibits a perfect three-fold symmetry, resembling a cloverleaf ( $65 \AA$ diameter) sitting on a short stem (30 A of length) (26). As shown in Fig. 1, SUN2 extends into the perinuclear space by its C-terminal SUN domain and interacts with nuclear lamina via its nucleoplasmic N-terminal domain $(11,27,28)$. Additionally, SUN2 connects with klarsicht/ANC-1/syne-1 homology (KASH) domain, providing mechanical transduction between the cytoskeleton and nuclear interior, directly $(19,22,28-30)$. Recent reports indicate that the SUN domain is at the center of a nucleocytoplasmic bridge that is essential for nuclear motility in cells (31). These observations suggest that the structural characteristics of SUN2 are crucial for nuclear anchoring, migration, and positioning $(19,22,29,30,32,33)$, centromere localization (34) and regulating the tethering of meiotic telomere (26). Thus, SUN2 may possess anti-cancer by regulating atypical nuclei structures in cancer cells.

\section{The role of SUN2 in different cancers}

Atypical teratoid/rhabdoid tumors (AT/RTs). AT/RT frequently occur in children. However, the pathogenesis of AT/RT remains to be uncovered. Several studies have indicated that the miR-221/222 gene cluster serves as an oncogenic miRNA in several types of human cancer $(35,36)$. Recently, miRNome and transcriptome traits in AT/RT were evaluated using small RNA sequencing and gene expression microarray analyses. Hsieh et al showed that miR-221/222-encoded miRNAs are abundantly expressed in AT/RT and substantially contribute to the malignancy of embryonal tumors (10). In AT/RT cells, overexpression of miR-221/222 leads to faster cell growth, and this observation is supported by previous reports that miR-221/222 promotes AT/RT malignancy and tumor growth in nude mice (14). AT/RT tissue microarray demonstrated that SUN2 is markedly decreased in AT/RT specimens. miRNAs generally execute their cellular functions through regulating target gene expression. Notably, miR-221/222 promotes cancer cell proliferation and tumor malignancy by targeting SUN2 mRNA in AT/RT, directly. Adherent cell growth of human medulloblastoma Daoy and human ATRT CHLA-02-ATRT cells was significantly increased upon transfection of SUN2 short hairpin-producing plasmids, parallelly, while overexpression of SUN2 reduced the proliferation rate (14). Together these studies show that SUN2 plays a critical role in miR-221/222-mediated AT/RT malignancy, indicating that SUN2 may be a promising target of AT/RT. For the first time, SUN2 was demonstrated closely relate to cancer initiation and progression.

Breast cancer. Several studies have provided evidence that abnormalities of the LINC are associated with complex alteration of biological processes and cancer occurrence. Reduced expression of lamin $\mathrm{A} / \mathrm{C}$ was detected in colon cancer (37), small cell lung cancer (38), leukemias and lymphomas $(39,40)$. However, lamin A/C is overexpressed in colorectal cancer (41), prostate cancer $(42)$, and skin cancer $(43,44)$. Therefore, the precise relationship between LINC complex components and the clinical significance of cancer still has not yet been well elucidated. In the present study, evaluation of four LINC complex and nuclear lamina components, SUN1, SUN2, nesprin-2, and lamin $\mathrm{A} / \mathrm{C}$, in breast cancer was performed. Matsumoto et al collected 73 breast cancer samples and found lower expression levels of LINC components in tumor regions compared with cancer-associated noncancerous regions (11). Furthermore, decreased expression of SUN2 was detected in 


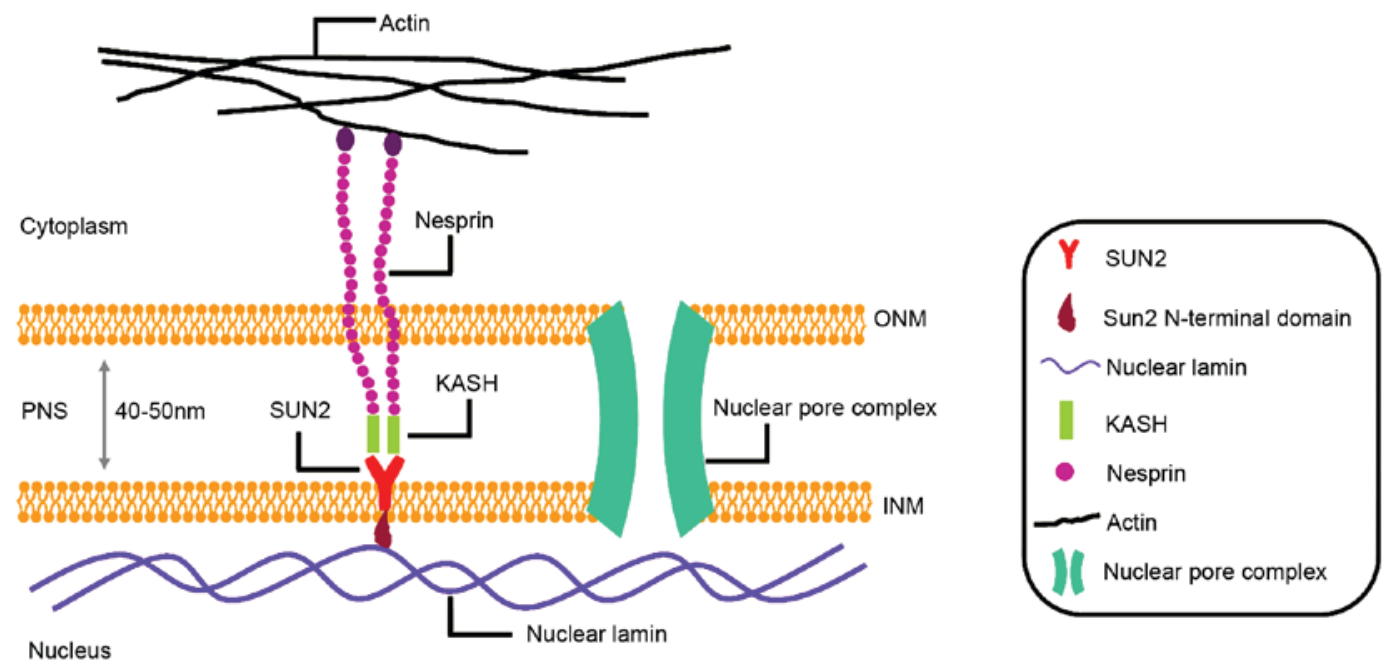

Figure 1. Schematic representation of SUN2 at the nuclear envelope. In the nuclear envelope, SUN2 exhibits a three-fold symmetry with its C-terminal extending into the perinuclear space and its nucleoplasmic N-terminal interacting with nuclear lamina. SUN2 connects with actin binding domain through binding to the KASH domain of Nesprin in cytoplasmic. The structure of SUN2 is functions as a bridge between the nuclear membrane and cytoplasm. SUN2, SAD1/UNC84 domain protein-2; KASH, klarsicht/ANC-1/syne-1 homology; PNS, perinuclear space; INM, inner nuclear membrane; ONM, outer nuclear membrane.

several breast cancer cell lines compared with noncancerous mammary gland cells in vitro. Together this demonstrates that the expression of SUN2 is attenuated in human breast cancer clinical specimens, indicating that SUN2 may have fundamental pathological functions in human breast cancer progression.

Lung cancer. Previous studies have demonstrated that SUN2 exhibits anti-cancer functions in lung cancer progression. Higher SUN2 expression predicts a better overall survival (OS) in lung cancer (12). Lv et al confirmed that expression level of SUN2 was significantly reduced in lung cancer tissues compared with paired normal tissues using Oncomine Database (12). According to the Protein Atlas Database, the expression of SUN2 is reduced in $75 \%$ (9 out of 12) of lung cancer tissue samples (12). Furthermore, in a previous study evaluating the relationship between SUN2 and lung cancer, lung cancer samples were subdivided into two groups and OS was analyzed. Individuals with lower SUN2 expression levels exhibited shorter OS than those with high SUN2 expression level (12). Together this indicates that downregulation of SUN2 in lung cancer progression and higher expression of SUN2 may predict a good outcome in human lung cancer occurrence. Additionally, ectopic expression of SUN2 inhibited lung cancer cell proliferation and colony formation abilities, and chemotherapy sensitivity to cisplatin treatment was increased when SUN2 was overexpressed in lung cancer cells. Further, knockdown of SUN2 promoted lung cancer cell proliferation and migration (12). Together these observations suggest that SUN2 is a key player in lung cancer development.

\section{SUN2 and DNA damage}

Cancers research remains a challenge to researchers, as genomic instability causes a constantly changing genetic profile of cancer occurrence (45). Failure of the DNA damage response (DDR) leads to genomic instability, which is one of enabling hallmarks that drive cancer occurrence (46). Phosphorylation of ataxia telangiectasia mutated (ATM) and H2A.X are among the earliest events in response to DNA damage (47-49). Recent studies showed that the expression level of $\gamma-\mathrm{H} 2 \mathrm{~A}$.X is significantly reduced in MEFs isolated from SUN1 ${ }^{--} \mathrm{SUN}^{-/-}$double knockout mice compared with wild-type mice. Although ATM is activated by $0.1 \mathrm{mM}$ of hydroxyurea (HU) in wild-type MEFs, ATM is not activated by $\mathrm{HU}$ in SUN1 $1^{--} \mathrm{SUN}^{-/-}$MEFs (16). There was no significant difference in tail moment between wild-type and $\mathrm{SUN}^{--}{ }^{-} \mathrm{SUN} 2^{--}$MEFs in the absence of methyl methane-sulfonate (16), which induces DNA damage (50). Interestingly, after treatment of SUN $1^{-/} \mathrm{SUN}^{-/-} \mathrm{MEFs}$ with methyl methane-sulfonate, a substantial increase occurred in the number of cells with prominent comet tails, indicative of DNA fragmentation (16). These observations reveal that DNA damage may accumulate rapidly in $\mathrm{SUN}^{--/} \mathrm{SUN}^{-1-}$ MEFs.

Phosphorylated checkpoint kinase-1 (Chk1), a cell-cycle checkpoint factor downstream of the DDR pathway, is reduced in SUN1 ${ }^{-1-} \mathrm{SUN}^{-/}$MEFs compared with wild-type mice (16). In addition, perinuclear heterochromatin is decreased in $\mathrm{SUN}^{--}{ }^{-} \mathrm{SUN} 2^{--}$MEFs, indicating that SUN1 and SUN2 participate in maintaining genomic stability, possibly by affecting DDR or DNA repair. Furthermore, MEFs from SUN1 ${ }^{--} \mathrm{SUN}^{-1-}$ mice exhibit a premature proliferative arrest at the $\mathrm{S}$ phase of cell cycle and increase in cell apoptosis $(16,51,52)$, leading to the death of $\mathrm{SUN}^{-/-} \mathrm{SUN}^{-1-}$ mice shortly after birth. These reports suggest that SUN2 may have crucial effects on evading cancer occurrence by its involvement in the DDR, and eliminating DNA lesions, maintaining genome stability and integrity.

SUN2 also interacts with DNA-PKcs that are potentially involved in the DDR, especially in DNA repair (16). Of note, cancer cells exhibit a high rate of proliferation and metabolic activities and DNA-PKcs plays an active part in regulation of cell proliferation (53). Of further interest, a previous study demonstrated that SUN2 exhibits suppression of cancer cell 
proliferation activity. In summary, uncovering the function of DNA-PKcs/SUN2 in regulation of cancer cells may offer potential avenues for cancers treatment.

\section{Overview of SUN2 signaling pathways in cancer}

MiR-221/222. miRNAs mainly function by regulating the expression of target genes at the post-transcriptional level. Up-regulation of miR-221/222 is associated with initiation and progression of breast cancer (54-59), liver cancer (60-63), pancreatic cancer (64-68), gastric cancer (69-73), colorectal cancer (74-78), glioma (79-85), multiple myeloma (86-89), and malignant melanoma $(90,91)$. TargetScan indicated a potentially favorable interaction between miR-221-3p/miR-222-3p and an 8-mer site at the position $255-262$ in the SUN2 3 '-untranslated region (3'UTR). Luciferase assays demonstrated that both miR-221-3p and miR-222-3p directly bind to the recognition element and reduce activity of Luc fused to full-length 3'UTR of SUN2 (14). Moreover, correlation coefficients (Pearson's $r$ ) between SUN2 and miR-221-3p as well as SUN2 and miR-222-3p are -0.777 and -0.802 , respectively, indicating negative correlation between SUN2 and miR-221/222 in AT/RT and medulloblastoma (MB) (14). A previous report also showed that the transcript and protein level of SUN2 was reduced after ectopic expression of miR-221/222, further supporting SUN2 as a direct target of miR-221/222. Increasing numbers of studies illustrate that expression of miR-221/222 induces cancer cell proliferation and invasion by inhibiting cancer suppressors and apoptotic genes (92). Over-expression of miR-221/222 significantly increases cell proliferation, while over-expression of both miR-221/222 and the complete coding sequence (CDS) of SUN2, which possesses no miR-221/222 recognition elements, counteracted the pro-proliferative effects (14). Together this suggests that one of the crucial pathways of miR-221/222 increasing cancer cell proliferation may occur by down-regulating SUN2 expression (Fig. 2).

SIRT5. Silent information regulator-5 (SIRT5) is a key component of the sirtuin family. SIRT5 expression has been associated with cancer prognosis and survival (93) via stimulating cancer cell proliferation and tumor growth, attenuating the tumor-type metabolism (94). Of note, the expression of SIRT5 is decreased in squamous cell carcinoma (95) and endometrial carcinoma (96). Thus, inhibition of SIRT5 may become a potential strategy to suppress the progression of cancers (97). Nevertheless, SIRT5 has also been found to have negative implications in certain types of malignancies (98). For instance, SIRT5 is highly expressed in human non-small cell lung cancer (NSCLC) and facilitates tumor growth and drug resistance (99). SIRT5 is also downregulated with histone deacetylase (HDACs) inhibitor treatment (100), while SUN2 expression dramatically increased in response to nicotinamide, an inhibitors of HDAC $(101,102)$, indicating that SUN2 may be regulated by SIRT5. Additionally, ectopic expression of SIRT5 significantly reduced SUN2 expression, while knockdown of SIRT5 dramatically increased SUN2 expression. Expression of SIRT5 inversely correlates with SUN2, indicating that SIRT5 acts as a negative regulator of SUN2, at least in part (12). The precise role

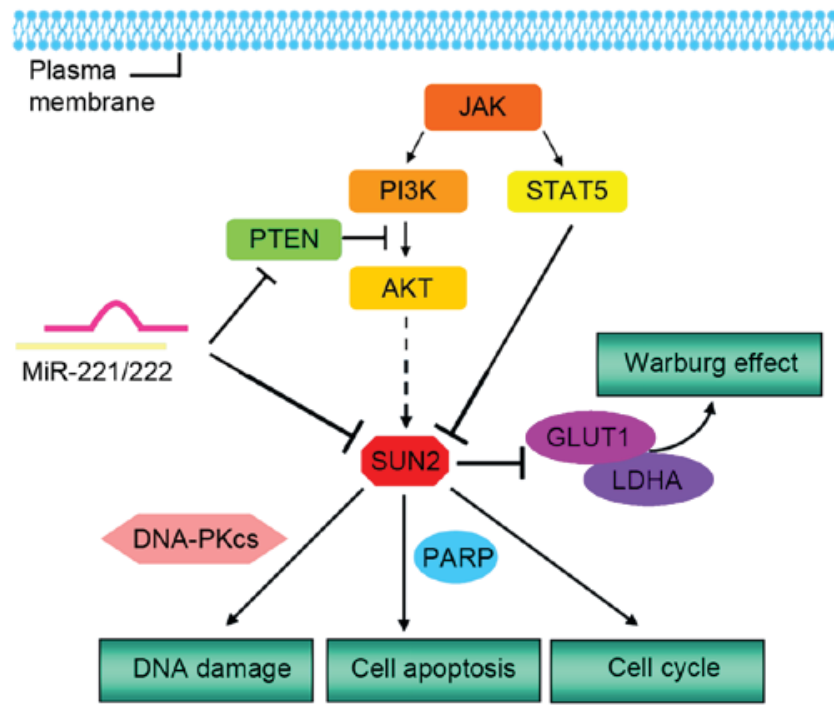

Figure 2. The potential target genes of SUN2 and SUN2-mediated effects implicated in cancer. The JAK signaling pathway-related PI3K/AKT and STAT pathways participate in the occurrence of cancers. Among the members of these pathways, STAT5 plays a role in the inhibition of SUN2. Ectopic expression of miR-221/222 reduces the expression of SUN2. Notably, SUN2 exhibits crucial functions in the cancer cell cycle, and SUN2 may exert effects on cancer cell apoptosis by regulating the cleavage of PARP Besides, SUN2 influences cancers progression by contacting GLUT1 and LDHA to attenuate the Warburg effect. During DNA damage response, SUN2 interacts with DNA-PKcs, which are involved in DNA repair and proliferation in cancer cells. JAK, janus protein tyrosine kinase; PTEN, phosphatase and tensin homology deleted on chromosome; PI3K; phosphatidylinositol 3-kinase; AKT, protein kinase B; SIRT5, silent information regulator-5; GLUT1, glucose transporter-1; LDHA, lactate dehydrogenase A; PARP, poly (ADP-ribose) polymerase; DNA-PKcs, DNA-dependent protein kinase catalytic subunit.

of SIRT5/SUN2 as a novel axis in the regulation of different types of cancers is currently unclear, and the relationship between SUN2 with cancer occurrence related to SIRT5 requires further exploration.

Warburg effect. Compared with normal cells, cancer cells exhibit a unique metabolism (103) to promote cell growth, survival, proliferation and long-term maintenance and fulfill the energetic demands of activities required for cancer cells (104). Studies have shown that the majority of cancer cells preferentially use aerobic glycolysis instead of oxidative phosphorylation to meet their increased energetic and biosynthetic demands $(105,106)$. This shifted metabolic patterning is known as the Warburg effect and is associated with cancer development $(106,107)$. Glucose transporter-1 (GLUT1) and lactate dehydrogenase A (LDHA), two key genes closely related to the Warburg effect, are needed for glucose uptake and conversion of pyruvate to lactate in cancer development (108). Recently, Recent studies demonstrated inverse correlations between SUN2 and GLUT1 as well as between SUN2 and LDAH. Ectopic expression SUN2 markedly decreased GLUT1 and LDHA expression levels, while knockdown of SUN2 increased expression of GLUT1 and LDHA (12). These results indicate that SUN2 may suppress cancer progression via attenuating the Warburg effect, at least in part. Though majority of cancer cells preferentially use aerobic glycolysis instead of oxidative phosphorylation to meet 
their increased energetic and biosynthetic demands $(105,106)$. Prostate cancer does not exhibit Warburg effect, an increase in glucose uptake (109). Herein, we cannot conclude that SUN2 exerts its anti-cancer effects in all types of cancers by inhibiting the Warburg effect.

PARP. Poly (ADP-ribose) polymerase (PARP) is verified as tightly correlated with cellular functions, such as DNA repair and transcriptional and posttranscriptional modulation of oncogenic gene expression, ultimately modulating carcinogenesis (110). Several studies suggested that PARP interacts with breast cancer (111), ovarian cancer (112), prostate cancer, lung cancer, gastric cancer and hepatocellular carcinoma $(113,114)$. Cleavage of PARP is a well-known marker of cell apoptosis, and interestingly, higher expression level of SUN2 increases PARP cleavage events (12). Together this suggests that SUN2 expression may inhibit cancer progression by regulating PARP-mediated cell apoptosis.

\section{Conclusion and prospective}

Overall, the complete underlying molecular mechanisms of cancers are still poorly elucidated. Many studies have established that dysregulations of oncogenes and cancer suppressor genes distinctly correlate with the initiation and progression of cancers. As outlined in this review, we discussed recent insights into the function of SUN2 in cancer progression. SUN2, as an anti-cancer member, participates in AT/RT, breast cancer and lung cancer by regulating biological processes in cancer cells, including cell cycle, apoptosis and migration. In addition, deficiency of SUN2 distinctly induces DNA damage, which is critically involved in cancer initiation.

Of note, SUN2 is widely expressed in different organs and tissues, such as the heart, brain, spleen, lung, liver, skeletal muscle, testis and embryos (17). Previous studies have shown an involvement of SUN2 in human cancers, such as cervical carcinoma, colorectal cancer, esophageal carcinoma and oral cavity squamous cell carcinoma. Furthermore, recent findings in fission yeast suggest that SUN2 may serve as a predictor and prognostic biomarkers in cancer. Therefore, we hypothesize that SUN2 may act as a potential biomarker in multiple cancer cell types. However, current findings suggest that SUN2 may present different functions in various cancers, and thus, we cannot definitively conclude that SUN2 solely functions as a cancer suppressor in all types of cancers. Undoubtedly, the precise functions and potential signaling pathways of SUN2 in cancer progression remain to be elucidated, and further studies and validations are urgently needed.

\section{Acknowledgements}

Not applicable.

\section{Funding}

This work is supported by the National Science Foundation of China (grant no. 81473268); the Anhui Provincial Higher Education Natural Science Foundation (grant no. KJ2016A364) and Anhui Provincial Natural Science Foundation (grant no. 21408085MKL31).

\section{Availability of data and materials}

Not applicable.

\section{Authors' contributions}

In this review, study concept and design: JL, XC, YC. Draft of the manuscript: XC, YC. Analysis of data: HMH, HDL, $\mathrm{CH}$, XMM. Critical revision of the manuscript for important intellectual content: FTB, XYP, YY, WXL, XFL. All authors agreed the final version.

\section{Ethics approval and consent to participate}

Not applicable.

\section{Consent for publication}

Not applicable.

\section{Competing interests}

The authors declare that they have no competing interests.

\section{References}

1. Lobo NA, Shimono Y, Qian D and Clarke MF: The biology of cancer stem cells. Annu Rev Cell Dev Biol 23: 675-699, 2007.

2. Peng XH, Huang HR, Lu J, Liu X, Zhao FP, Zhang B, Lin SX, Wang L, Chen HH, Xu X, et al: MiR-124 suppresses tumor growth and metastasis by targeting Foxq1 in nasopharyngeal carcinoma. Mol Cancer 13: 186, 2014.

3. Liu Y, Li Z, Wu L, Wang Z, Wang X, Yu Y, Zhao Q and Luo F: MiRNA-125a-5p: A regulator and predictor of gefitinib's effect on nasopharyngeal carcinoma. Cancer Cell Int 14: 24, 2014.

4. Liu X, Lv XB, Wang XP, Sang Y, Xu S, Hu K, Wu M, Liang Y, Liu P, Tang J, et al: MiR-138 suppressed nasopharyngeal carcinoma growth and tumorigenesis by targeting the CCND1 oncogene. Cell Cycle 11: 2495-2506, 2012.

5. Qi X, Li J, Zhou C, Lv C and Tian M: MicroRNA-320a inhibits cell proliferation, migration and invasion by targeting BMI-1 in nasopharyngeal carcinoma. FEBS Lett 588: 3732-3738, 2014

6. Cheung CC, Chung GT, Lun SW, To KF, Choy KW, Lau KM, Siu SP, Guan XY, Ngan RK, Yip TT, et al: miR-31 is consistently inactivated in EBV-associated nasopharyngeal carcinoma and contributes to its tumorigenesis. Mol Cancer 13: 184, 2014.

7. Liu X, Yu X, Xie J, Zhan M, Yu Z, Xie L, Zeng H, Zhang F, Chen G, Yi X and Zheng J: ANGPTL2/LILRB2 signaling promotes the propagation of lung cancer cells. Oncotarget 6: 21004-21015, 2015.

8. Wang J, Tian X, Han R, Zhang X, Wang X, Shen H, Xue L, Liu Y, Yan X, Shen J, et al: Downregulation of miR-486-5p contributes to tumor progression and metastasis by targeting protumorigenic ARHGAP5 in lung cancer. Oncogene 33: 1181-1189, 2014.

9. Hecht I, Natan S, Zaritsky A, Levine H, Tsarfaty I and Ben-Jacob E: The motility-proliferation-metabolism interplay during metastatic invasion. Sci Rep 5: 13538, 2015.

10. Hsieh TH, Chien CL, Lee YH, Lin CI, Hsieh JY, Chao ME, Liu DJ, Chu SS, Chen W, Lin SC, et al: Downregulation of SUN2, a novel tumor suppressor, mediates miR-221/222-induced malignancy in central nervous system embryonal tumors. Carcinogenesis 35: 2164-2174, 2014.

11. Matsumoto A, Hieda M, Yokoyama Y, Nishioka Y, Yoshidome K, Tsujimoto M and Matsuura N: Global loss of a nuclear lamina component, lamin A/C, and LINC complex components SUN1, SUN2, and nesprin-2 in breast cancer. Cancer Med 4: 1547-1557, 2015. 
12. Lv XB, Liu L, Cheng C, Yu B, Xiong L, Hu K, Tang J, Zeng L and Sang Y: SUN2 exerts tumor suppressor functions by suppressing the Warburg effect in lung cancer. Sci Rep 5: 17940, 2015.

13. Meinke P, Nguyen TD and Wehnert MS: The LINC complex and human disease. Biochem Soc Trans 39: 1693-1697, 2011.

14. Khatau SB, Hale CM, Stewart-Hutchinson PJ, Patel MS, Stewart CL, Searson PC, Hodzic D and Wirtz D: A perinuclear actin cap regulates nuclear shape. Proc Natl Acad Sci USA 106: 19017-19022, 2009.

15. Wang Z, Zhu WG and Xu X: Ubiquitin-like modifications in the DNA damage response. Mutat Res 803-805: 56-75, 2017.

16. Lei K, Zhu X, Xu R, Shao C, Xu T, Zhuang Y and Han M: Inner nuclear envelope proteins SUN1 and SUN2 play a prominent role in the DNA damage response. Curr Biol 22: 1609-1615, 2012.

17. Wang Q, Du X, Cai Z and Greene MI: Characterization of the structures involved in localization of the SUN proteins to the nuclear envelope and the centrosome. DNA Cell Biol 25: 554-562, 2006

18. Hodzic DM, Yeater DB, Bengtsson L, Otto H and Stahl PD: Sun2 is a novel mammalian inner nuclear membrane protein. J Biol Chem 279: 25805-25812, 2004.

19. Padmakumar VC, Libotte T, Lu W, Zaim H, Abraham S, Noegel AA, Gotzmann J, Foisner R and Karakesisoglou I: The inner nuclear membrane protein Sun1 mediates the anchorage of Nesprin-2 to the nuclear envelope. J Cell Scie 118: 3419-3430, 2005.

20. Dreger M, Bengtsson L, Schöneberg T, Otto H and Hucho F: Nuclear envelope proteomics: Novel integral membrane proteins of the inner nuclear membrane. Proc Natl Acad Sci USA 98 11943-11948, 2001.

21. Kennedy C, Sebire K, de Kretser DM and O'Bryan MK: Human sperm associated antigen 4 (SPAG4) is a potential cancer marker. Cell Tissue Res 315: 279-283, 2004.

22. Tzur YB, Wilson KL and Gruenbaum Y: SUN-domain proteins: 'Velcro' that links the nucleoskeleton to the cytoskeleton. Nat Rev Mol Cell Biol 7: 782-788, 2006.

23. Starr DA and Fischer JA: KASH 'n Karry: The KASH domain family of cargo-specific cytoskeletal adaptor proteins. Bioessays 27: 1136-1146, 2005.

24. Hagan I and Yanagida M: The product of the spindle formation gene sad1+ associates with the fission yeast spindle pole body and is essential for viability. J Cell Biol 129: 1033-1047, 1995.

25. Malone CJ, Fixsen WD, Horvitz HR and Han M: UNC-84 localizes to the nuclear envelope and is required for nuclear migration and anchoring during C. elegans development. Development 126 : 3171-3181, 1999.

26. Zhou Z, Du X, Cai Z, Song X, Zhang H, Mizuno T, Suzuki E, Yee MR, Berezov A, Murali R, et al: Structure of Sad1-UNC84 homology (SUN) domain defines features of molecular bridge in nuclear envelope. J Biol Chem 287: 5317-5326, 2012.

27. Starr DA and Fridolfsson HN: Interactions between nuclei and the cytoskeleton are mediated by SUN-KASH nuclear-envelope bridges. Annu Rev Cell Dev Biol 26: 421-444, 2010.

28. Stewart-Hutchinson PJ, Hale CM, Wirtz D and Hodzic D: Structural requirements for the assembly of LINC complexes and their function in cellular mechanical stiffness. Exp Cell Res 314: 1892-1905, 2008.

29. Crisp M, Liu Q, Roux K, Rattner JB, Shanahan C, Burke B, Stahl PD and Hodzic D: Coupling of the nucleus and cytoplasm: Role of the LINC complex. J Cell Biol 172: 41-53, 2006.

30. Haque F, Lloyd DJ, Smallwood DT, Dent CL, Shanahan CM, Fry AM, Trembath RC and Shackleton S: SUN1 interacts with nuclear lamin A and cytoplasmic nesprins to provide a physical connection between the nuclear lamina and the cytoskeleton. Mol Cell Biol 26: 3738-3751, 2006.

31. Schmitt J, Benavente R, Hodzic D, Höög C, Stewart CL and Alsheimer M: Transmembrane protein Sun2 is involved in tethering mammalian meiotic telomeres to the nuclear envelope. Proc Natl Acad Sci USA 104: 7426-7431, 2007.

32. Lee KK, Starr D, Cohen M, Liu J, Han M, Wilson KL and Gruenbaum Y: Lamin-dependent localization of UNC-84, a protein required for nuclear migration in Caenorhabditis elegans. Mol Biol Cell 13: 892-901, 2002.

33. Starr DA and Han M: ANChors away: An actin based mechanism of nuclear positioning. J Cell Scie 116: 211-216, 2003.

34. Ostlund C, Folker ES, Choi JC, Gomes ER, Gundersen GG and Worman HJ: Dynamics and molecular interactions of linker of nucleoskeleton and cytoskeleton (LINC) complex proteins. J Cell Sci 122: 4099-4108, 2009
35. Chistiakov DA, Sobenin IA, Orekhov AN and Bobryshev YV: Human miR-221/222 in physiological and atherosclerotic vascular remodeling. Biomed Res Int 2015: 354517, 2015.

36. Song J, Ouyang Y, Che J, Li X, Zhao Y, Yang K, Zhao X, Chen Y, Fan C and Yuan W: Potential value of miR-221/222 as diagnostic, prognostic and therapeutic biomarkers for diseases. Front Immunol 8: 56, 2017.

37. Wu Z, Wu L, Weng D, Xu D, Geng J and Zhao F: Reduced expression of lamin $\mathrm{A} / \mathrm{C}$ correlates with poor histological differentiation and prognosis in primary gastric carcinoma. J Exp Clin Cancer Res 28: 8, 2009.

38. Broers JL, Raymond Y, Rot MK, Kuijpers H, Wagenaar SS and Ramaekers FC: Nuclear A-type lamins are differentially expressed in human lung cancer subtypes. Am J Pathol 143: 211-220, 1993.

39. Stadelmann B, Khandjian E, Hirt A, Lüthy A, Weil R and Wagner HP: Repression of nuclear lamin A and C gene expression in human acute lymphoblastic leukemia and non-Hodgkin's lymphoma cells. Leuk Res 14: 815-821, 1990.

40. Agrelo R, Setien F, Espada J, Artiga MJ, Rodriguez M, Pérez-Rosado A, Sanchez-Aguilera A, Fraga MF, Piris MA and Esteller M: Inactivation of the lamin $\mathrm{A} / \mathrm{C}$ gene by $\mathrm{CpG}$ island promoter hypermethylation in hematologic malignancies, and its association with poor survival in nodal diffuse large B-cell lymphoma. J Clin Oncol 23: 3940-3947, 2005.

41. Willis ND, Cox TR, Rahman-Casañs SF, Smits K, Przyborski SA, van den Brandt P, van Engeland M, Weijenberg M, Wilson RG, de Bruïne $\mathrm{A}$ and Hutchison CJ: Lamin $\mathrm{A} / \mathrm{C}$ is a risk biomarker in colorectal cancer. PLoS One 3: e2988, 2008.

42. Kong L, Schäfer G, Bu H, Zhang Y and Klocker H: Lamin A/C protein is overexpressed in tissue-invading prostate cancer and promotes prostate cancer cell growth, migration and invasion through the PI3K/AKT/PTEN pathway. Carcinogenesis 33: 751-759, 2012.

43. Tilli CM, Ramaekers FC, Broers JL, Hutchison CJ and Neumann HA: Lamin expression in normal human skin, actinic keratosis, squamous cell carcinoma and basal cell carcinoma. $\mathrm{Br}$ J Dermatol 148: 102-109, 2003.

44. Venables RS, McLean S, Luny D, Moteleb E, Morley S, Quinlan RA, Lane EB and Hutchison CJ: Expression of individual lamins in basal cell carcinomas of the skin. Br J Cancer 84: 512-519, 2001.

45. Ellenbroek SI and van Rheenen J: Imaging hallmarks of cancer in living mice. Nat Rev Cancer 14: 406-418, 2014.

46. Hanahan D and Weinberg RA: Hallmarks of cancer: The next generation. Cell 144: 646-674, 2011

47. Ciccia A and Elledge SJ: The DNA damage response: Making it safe to play with knives. Mol Cell 40: 179-204, 2010

48. Paull TT, Rogakou EP, Yamazaki V, Kirchgessner CU, Gellert M and Bonner WM: A critical role for histone $\mathrm{H} 2 \mathrm{AX}$ in recruitment of repair factors to nuclear foci after DNA damage. Curr Biol 10: 886-895, 2000

49. Harper JW and Elledge SJ: The DNA damage response: Ten years after. Mol Cell 28: 739-745, 2007.

50. Sobol RW, Horton JK, Kühn R, Gu H, Singhal RK, Prasad R, Rajewsky K and Wilson SH: Requirement of mammalian DNA polymerase-beta in base-excision repair. Nature 379: 183-186, 1996.

51. Majidinia M and Yousefi B: DNA repair and damage pathways in breast cancer development and therapy. DNA Repair (Amst) 54: 22-29, 2017

52. Zhang X, Lei K, Yuan X, Wu X, Zhuang Y, Xu T, Xu R and Han M: SUN1/2 and Syne/Nesprin-1/2 complexes connect centrosome to the nucleus during neurogenesis and neuronal migration in mice. Neuron 64: 173-187, 2009.

53. Davidson D, Amrein L, Panasci L and Aloyz R: Small molecules, inhibitors of DNA-PK, targeting DNA repair, and beyond. Front Pharmacol 4: 5, 2013.

54. Stinson S, Lackner MR, Adai AT, Yu N, Kim HJ, O'Brien C, Spoerke J, Jhunjhunwala S, Boyd Z, Januario T, et al: miR-221/222 targeting of trichorhinophalangeal 1 (TRPS1) promotes epithelial-to-mesenchymal transition in breast cancer. Sci Signal 4: pt5, 2011

55. Hwang MS, Yu N, Stinson SY, Yue P, Newman RJ, Allan BB and Dornan D: miR-221/222 targets adiponectin receptor 1 to promote the epithelial-to-mesenchymal transition in breast cancer. PLoS One 8: e66502, 2013.

56. Li Y, Liang C, Ma H, Zhao Q, Lu Y, Xiang Z, Li L, Qin J, Chen Y, Cho WC, et al: miR-221/222 promotes S-phase entry and cellular migration in control of basal-like breast cance. Molecules 19: 7122-7137, 2014 
57. Gan R, Yang Y, Yang $X$, Zhao $L$, Lu J and Meng QH Downregulation of miR-221/222 enhances sensitivity of breast cancer cells to tamoxifen through upregulation of TIMP3. Cancer Gene Ther 21: 290-296, 2014.

58. Pichiorri F, Palmieri D, De Luca L, Consiglio J, You J, Rocci A, Talabere T, Piovan C, Lagana A, Cascione L, et al: In vivo NCL targeting affects breast cancer aggressiveness through miRNA regulation. J Exp Med 210: 951-968, 2013.

59. Falkenberg N, Anastasov N, Rappl K, Braselmann H, Auer G, Walch A, Huber M, Höfig I, Schmitt M, Höfler H, et al: MiR-221/-222 differentiate prognostic groups in advanced breast cancers and influence cell invasion. Br J Cancer 109: 2714-2723, 2013.

60. Gramantieri L, Fornari F, Ferracin M, Veronese A, Sabbioni S, Calin GA, Grazi GL, Croce CM, Bolondi L and Negrini M: MicroRNA-221 targets Bmf in hepatocellular carcinoma and correlates with tumor multifocality. Clin Cancer Res 15: 5073-5081, 2009.

61. Bae HJ, Jung KH, Eun JW, Shen Q, Kim HS, Park SJ, Shin WC, Yang HD, Park WS, Lee JY and Nam SW: MicroRNA-221 governs tumor suppressor HDAC6 to potentiate malignant progression of liver cancer. J Hepatol 63: 408-419, 2015.

62. Callegari E, Elamin BK, Giannone F, Milazzo M, Altavilla G, Fornari F, Giacomelli L, D'Abundo L, Ferracin M, Bassi C, et al: Liver tumorigenicity promoted by microRNA-221 in a mouse transgenic model. Hepatology 56: 1025-1033, 2012.

63. Li J, Wang Y, Yu W, Chen J and Luo J: Expression of serum miR-221 in human hepatocellular carcinoma and its prognostic significance. Biochem Biophys Res Commun 406: 70-73, 2011.

64. Duan M, Yao H, Hu G, Chen X, Lund AK and Buch S: HIV Tat induces expression of ICAM-1 in HUVECs: implications for miR-221/-222 in HIV-associated cardiomyopathy. PLoS One 8: e60170, 2013

65. Sarkar S, Dubaybo H, Ali S, Goncalves P, Kollepara SL, Sethi S, Philip PA and Li Y: Down-regulation of miR-221 inhibits proliferation of pancreatic cancer cells through up-regulation of PTEN, p27(kip1), p57(kip2), and PUMA. Am J Cancer Res 3: 465-477, 2013.

66. Passadouro M, Pedroso de Lima MC and Faneca H: MicroRNA modulation combined with sunitinib as a novel therapeutic strategy for pancreatic cancer. Int J Nanomedicine 9: 3203-3217, 2014.

67. Tanaka R, Tomosugi M, Horinaka M, Sowa Y and Sakai T: Metformin causes G1-phase arrest via down-regulation of MiR-221 and enhances TRAIL sensitivity through DR5 Up-regulation in pancreatic cancer cells. PLoS One 10: e0125779, 2015.

68. Lee C, He H, Jiang Y, Di Y, Yang F, Li J, Jin C and Fu D: Elevated expression of tumor miR-222 in pancreatic cancer is associated with Ki67 and poor prognosis. Med Oncol 30: 700, 2013.

69. Kim YK, Yu J, Han TS, Park SY, Namkoong B, Kim DH Hur K, Yoo MW, Lee HJ, Yang HK and Kim VN: Functional links between clustered microRNAs: suppression of cell-cycle inhibitors by microRNA clusters in gastric cancer. Nucleic Acids Res 37: 1672-1681, 2009.

70. Liu W, Song N, Yao H, Zhao L, Liu H and Li G: miR-221 and miR-222 simultaneously target RECK and regulate growth and invasion of gastric cancer cells. Med Sci Monit 21: 2718-2725, 2015.

71. Chun-Zhi Z, Lei H, An-Ling Z, Yan-Chao F, Xiao Y, Guang-Xiu W, Zhi-Fan J, Pei-Yu P, Qing-Yu Z and Chun-Sheng K: MicroRNA-221 and microRNA-222 regulate gastric carcinoma cell proliferation and radioresistance by targeting PTEN. BMC Cancer 10:367, 2010.

72. Song MY, Pan KF, Su HJ, Zhang L, Ma JL, Li JY, Yuasa Y, Kang D, Kim YS and You WC: Identification of serum microRNAs as novel non-invasive biomarkers for early detection of gastric cancer. PLoS One 7: e33608, 2012.

73. Fu Z, Qian F, Yang X, Jiang H, Chen Y and Liu S: Circulating miR-222 in plasma and its potential diagnostic and prognostic value in gastric cancer. Med Oncol 31: 164, 2014.

74. Sun K, Wang W, Zeng JJ, Wu CT, Lei ST and Li GX: MicroRNA-221 inhibits CDKN1C/p57 expression in human colorectal carcinoma. Acta Pharmacol Sin 32: 375-384, 2011.

75. Qin J and Luo M: MicroRNA-221 promotes colorectal cancer cell invasion and metastasis by targeting RECK. FEBS Lett 588: 99-104, 2014

76. Liu S, Sun X, Wang M, Hou Y, Zhan Y, Jiang Y, Liu Z, Cao X, Chen P, Liu Z et al: A microRNA 221- and 222-mediated feedback loop maintains constitutive activation of $\mathrm{NF}_{\kappa \mathrm{B}}$ and STAT3 in colorectal cancer cells. Gastroenterology 147: 847-859 e811, 2014.
77. Xue Q, Sun K, Deng HJ, Lei ST, Dong JQ and Li GX: Anti-miRNA-221 sensitizes human colorectal carcinoma cells to radiation by upregulating PTEN. World J Gastroenterol 19: 9307-9317, 2013

78. Pu XX, Huang GL, Guo HQ, Guo CC, Li H, Ye S, Ling S, Jiang L, Tian Y and Lin TY: Circulating miR-221 directly amplified from plasma is a potential diagnostic and prognostic marker of colorectal cancer and is correlated with p53 expression. J Gastroenterol Hepatol 25: 1674-1680, 2010.

79. Zhang C, Zhang J, Hao J, Shi Z, Wang Y, Han L, Yu S, You Y, Jiang $\mathrm{T}$, Wang J, et al: High level of miR-221/222 confers increased cell invasion and poor prognosis in glioma. J Transl Med 10: 119, 2012.

80. Medina R, Zaidi SK, Liu CG, Stein JL, van Wijnen AJ, Croce CM and Stein GS: MicroRNAs 221 and 222 bypass quiescence and compromise cell survival. Cancer Res 68: 2773-2780, 2008.

81. Zhang C, Kang C, You Y, Pu P, Yang W, Zhao P, Wang G, Zhang A, Jia Z, Han L and Jiang H: Co-suppression of miR-221/222 cluster suppresses human glioma cell growth by targeting p27kip1 in vitro and in vivo. Int J Oncol 34: 1653-1660, 2009.

82. Zhang CZ, Zhang JX, Zhang AL, Shi ZD, Han L, Jia ZF, Yang WD, Wang GX, Jiang T, You YP, et al: MiR-221 and miR-222 target PUMA to induce cell survival in glioblastoma. Mol Cancer 9: 229, 2010.

83. Quintavalle C, Garofalo M, Zanca C, Romano G, Iaboni M, del Basso De Caro M, Martinez-Montero JC, Incoronato M, Nuovo G, Croce CM and Condorelli G: miR-221/222 overexpession in human glioblastoma increases invasiveness by targeting the protein phosphate $\mathrm{PTP} \mu$. Oncogene 31: 858-868, 2012

84. Chen L, Zhang J, Han L, Zhang A, Zhang C, Zheng Y, Jiang T, $\mathrm{Pu}$ P, Jiang C and Kang C: Downregulation of miR-221/222 sensitizes glioma cells to temozolomide by regulating apoptosis independently of p53 status. Oncol Rep 27: 854-860, 2012.

85. Li W, Guo F, Wang P, Hong S and Zhang C: miR-221/222 confers radioresistance in glioblastoma cells through activating Akt independent of PTEN status. Curr Mol Med 14: 185-195, 2014.

86. Di Martino MT, Gullà A, Cantafio ME, Lionetti M, Leone E, Amodio N, Guzzi PH, Foresta U, Conforti F, Cannataro M, et al: In vitro and in vivo anti-tumor activity of miR-221/222 inhibitors in multiple myeloma. Oncotarget 4: 242-255, 2013

87. Di Martino MT, Gullà A, Gallo Cantafio ME, Altomare E, Amodio N, Leone E, Morelli E, Lio SG, Caracciolo D, Rossi M, et al: In vitro and in vivo activity of a novel locked nucleic acid (LNA)-inhibitor-miR-221 against multiple myeloma cells. PLoS One 9: e89659, 2014

88. Gullà A, Di Martino MT, Gallo Cantafio ME, Morelli E, Amodio N, Botta C, Pitari MR, Lio SG, Britti D, Stamato MA, et al: A 13 mer LNA-i-miR-221 inhibitor restores drug sensitivity in melphalan-refractory multiple myeloma cells Clin Cancer Res 22: 1222-1233, 2016.

89. Huang JJ, Yu J, Li JY, Liu YT and Zhong RQ: Circulating microRNA expression is associated with genetic subtype and survival of multiple myeloma. Med Oncol 29: 2402-2408, 2012.

90. Kanemaru H, Fukushima S, Yamashita J, Honda N, Oyama R, Kakimoto A, Masuguchi S, Ishihara T, Inoue Y, Jinnin M and Ihn H: The circulating microRNA-221 level in patients with malignant melanoma as a new tumor marker. J Dermatol Sci 61: 187-193, 2011.

91. Felicetti F, De Feo A, Coscia C, Puglisi R, Pedini F, Pasquini L, Bellenghi M,Errico MC,PaganiE and Carè A: Exosome-mediated transfer of miR-222 is sufficient to increase tumor malignancy in melanoma. J Transl Med 14: 56, 2016.

92. Alamolhodaei NS, Behravan J, Mosaffa F and Karimi G: MiR 221/222 as new players in tamoxifen resistance. Curr Pharm Des 22: 6946-6955, 2016.

93. Linher-Melville K and Singh G: The complex roles of STAT3 and STAT5 in maintaining redox balance: Lessons from STAT-mediated xCT expression in cancer cells. Mol Cell Endocrinol 451: 40-52, 2017.

94. Li F, He X, Ye D, Lin Y, Yu H, Yao C, Huang L, Zhang J, Wang F, $\mathrm{Xu}$ S, et al: NADP(+)-IDH mutations promote hypersuccinylation that impairs mitochondria respiration and induces apoptosis resistance. Mol Cell 60: 661-675, 2015.

95. Lai CC, Lin PM, Lin SF, Hsu CH, Lin HC, Hu ML, Hsu CM amd Yang MY: Altered expression of SIRT gene family in head and neck squamous cell carcinoma. Tumour Biol 34: 1847-1854, 2013

96. Kim DH, Kwak Y, Kim ND and Sim T: Antitumor effects and molecular mechanisms of ponatinib on endometrial cancer cells harboring activating FGFR2 mutations. Cancer Biol Ther 17: $65-78,2016$. 
97. Xiangyun Y, Xiaomin N, Linping G, Yunhua X, Ziming L, Yongfeng Y, Zhiwei C and Shun L: Desuccinylation of pyruvate kinase M2 by SIRT5 contributes to antioxidant response and tumor growth. Oncotarget 8: 6984-6993, 2017.

98. Osborne B, Bentley NL, Montgomery MK and Turner N: The role of mitochondrial sirtuins in health and disease. Free Radic Biol Med 100: 164-174, 2016.

99. Lu W, Zuo Y, Feng Y and Zhang M: SIRT5 facilitates cancer cell growth and drug resistance in non-small cell lung cancer. Tumour Biol 35: 10699-10705, 2014.

100. Kyrylenko S, Kyrylenko O, Suuronen T and Salminen A: Differential regulation of the Sir2 histone deacetylase gene family by inhibitors of class I and II histone deacetylases. Cell Mol Life Sci 60: 1990-1997, 2003.

101. Ding S, Khoury-Hanold W, Iwasaki A and Robek MD: Epigenetic reprogramming of the type III interferon response potentiates antiviral activity and suppresses tumor growth. PLoS Biol 12: e1001758, 2014.

102. Vanhaecke T, Papeleu P, Elaut G and Rogiers V: Trichostatin A-like hydroxamate histone deacetylase inhibitors as therapeutic agents: toxicological point of view. Curr Med Chem 11: 1629-1643, 2004.

103. Zhou W, Liotta LA and Petricoin EF: The Warburg effect and mass spectrometry-based proteomic analysis. Cancer Genomics Proteomics 14: 211-218, 2017.

104. Sica A, Strauss L, Consonni FM, Travelli C, Genazzani A and Porta C: Metabolic regulation of suppressive myeloid cells in cancer. Cytokine Growth Factor Rev 35: 27-35, 2017.

105. Cairns RA: Drivers of the Warburg phenotype. Cancer J 21: 56-61, 2015

106. He X, Li C, Ke R, Luo L and Huang D: Down-regulation of adenosine monophosphate-activated protein kinase activity: A driver of cancer. Tumour Biol 39: 1010428317697576, 2017.
107. Yang W, Zheng Y, Xia Y, Ji H, Chen X, Guo F, Lyssiotis CA, Aldape K, Cantley LC and Lu Z: ERK1/2-dependent phosphorylation and nuclear translocation of PKM2 promotes the Warburg effect. Nat Cell Biol 14: 1295-1304, 2012.

108. Liu J, Zhang C, Wu R, Lin M, Liang Y, Liu J, Wang X, Yang B and Feng Z: RRAD inhibits the Warburg effect through negative regulation of the NF- $\kappa \mathrm{B}$ signaling. Oncotarget 6: 14982-14992, 2015.

109. Dueregger A, Schöpf B, Eder T, Höfer J, Gnaiger E, Aufinger A, Kenner L, Perktold B, Ramoner R, Klocker H and Eder IE: Differential utilization of dietary fatty acids in benign and malignant cells of the prostate. PLoS One 10: e0135704, 2015.

110. He JX, Yang CH and Miao ZH: Poly(ADP-ribose) polymerase inhibitors as promising cancer therapeutics. Acta Pharmacol Sin 31: 1172-1180, 2010.

111. Livraghi L and Garber JE: PARP inhibitors in the management of breast cancer: current data and future prospects. BMC Med 13: 188, 2015.

112. Evans T and Matulonis U: PARP inhibitors in ovarian cancer: Evidence, experience and clinical potential. Ther Adv Med Oncol 9: 253-267, 2017.

113. Rajawat J, Shukla N and Mishra DP: Therapeutic targeting of poly(ADP-Ribose) polymerase-1 (PARP1) in cancer: Current developments, therapeutic strategies, and future opportunities. Med Res Rev 37: 1461-1491, 2017.

114. Vici P, Mariani L, Pizzuti L, Sergi D, Di Lauro L, Vizza E, Tomao F, Tomao S, Mancini E, Vincenzoni C, et al: Emerging biological treatments for uterine cervical carcinoma. J Cancer 5 : 86-97, 2014

(c) (i) This work is licensed under a Creative Commons Attribution 4.0 International (CC BY 4.0) License. 